“C 2014 IEEE. Personal use of this material is permitted. Permission from IEEE must be obtained for all other uses, in any current or future media, including reprinting/republishing this material for advertising or promotional purposes, creating new collective works, for resale or redistribution to servers or lists, or reuse of any copyrighted component of this work in other works." 


\title{
High Directivity, Lens-less THz Photoconductive Switch Dipole Antennas
}

\author{
Ning Zhu ${ }^{1}$ and Richard W. Ziolkowski ${ }^{2}$ \\ ${ }^{1}$ RF Group, Freescale Semiconductor Inc., Tempe, AZ, USA, nzhu@email.arizona.edu \\ ${ }^{2}$ Department of Electrical and Computer Engineering, University of Arizona, Tucson, AZ, USA, ziolkowski@ece.arizona.edu
}

\begin{abstract}
We present several linearly polarized terahertz (THz) photoconductive switch antennas that have been designed for a compressive sensing-based $\mathrm{THz}$ imaging system which is currently being brought online. A bow-tie based antenna, including a finite ground plane (on the side of the substrate opposite the bow-tie) and its DC bias lines, is the reference design. By incorporating a metamaterial-inspired artificial magnetic conductor (AMC) structure, the directivity is increased by almost $3 \mathrm{~dB}$ at $1.05 \mathrm{THz}$. The simulation results show that the radiation efficiency of this bow-tie AMC-augmented antenna is above $85 \%$ at $1.05 \mathrm{THz}$. A single capacitively-loaded dipole antenna is then introduced; it has a $12.8 \mathrm{~dB}$ directivity, a $11.6 \mathrm{~dB}$ realized gain, and a $82 \%$ radiation efficiency. By connecting two single capacitively-loaded dipole antennas to form a linear array, higher directivity (14.8 dB), lower sidelobe level $(-18 \mathrm{~dB})$ and larger front-to-back-ratio $(14 \mathrm{~dB})$ values are achieved. Additional designs will be given in the presentation, including the incorporation of a meta-film structure to decrease the sidelobe levels and increase the front-to-back ratio.
\end{abstract}

Index Terms-Directivity, Input impedance, Photoconductive antennas, Directivity patterns, THz antennas.

\section{INTRODUCTION}

Because $\mathrm{THz}$ imaging systems are able to provide images with higher resolution than microwave imaging systems and are non-ionizing so they also offer less potential harm to the human body than X-ray imaging systems, they have drawn considerable attention recently, for instance, in quality control, non-destructive evaluation, medical, and security applications. As one of the most important components in a $\mathrm{THz}$ imaging system, the antenna plays both impedance matching and power radiating roles. Some representative $\mathrm{THz}$ antenna examples can be found in [1-4]. While both photoconductive and photomixer style $\mathrm{THz}$ sources continue to be considered by the $\mathrm{THz}$ community, we emphasize the former in all of the designs reported here.

A single capacitively-loaded $\mathrm{THz}$ dipole antenna is initially designed to achieve the high directivity and high radiation efficiency properties desired for eventual application in a $\mathrm{THz}$ spectral imaging system. The capacitively-loaded structure also incorporates the necessary DC bias and ground lines. By connecting the DC bias and ground lines of two single capacitively-loaded $\mathrm{THz}$ dipole antennas together, an antenna array is generated. The impedance matching and performance characteristics of the array are fine tuned to achieve even more appealing radiation properties. In addition, the impact of the introduction of a meta-film structure-based superstrate into this array also will be given in our presentation. Since the aperture efficiencies for the original capacitively-loaded $\mathrm{THz}$ dipole antenna and the corresponding array are already over $100 \%$, there is not much room to increase the directivity. Nevertheless, the presence of the superstrate provides additional design degrees of freedom which allow the increase of the front-to-back ratio value of the array at a trade-off cost of slightly increasing the side lobe levels of its radiation patterns. All the antennas in this paper were simulated with ANSYS/ANSOFT's high frequency structure simulator (HFSS).

\section{BOWTIE-SHAPED THZ ANTENNA DESIGN}

The bowtie-shaped $\mathrm{THz}$ antenna used as the reference case is shown in Fig. 1. A $0.35-\mu \mathrm{m}$-thick Ti-Au $\left(\sigma=1.6 \times 10^{7} \mathrm{~S} / \mathrm{m}\right)$ film was selected for the antenna and the ground plane; a 1$\mu \mathrm{m}$-thick low-temperature-grown (LTG) GaAs substrate was placed on top of a $59-\mu$ m-thick GaAs $\left(\varepsilon_{\mathrm{r}}=12.9, \mu_{\mathrm{r}}=1.0\right.$, loss tangent $=0.006$ ). To further increase the $10 \mathrm{~dB}$ bandwidth of the antenna, the edges of the bowtie structure were cut in a circular arc (radius $76.3 \mathrm{um}$ ) rather than in a straight line. A vertical cylinder with $56-\mu \mathrm{m}$ height and $1-\mu \mathrm{m}$ radius was cut through the ground plane and partially through the substrate for the laser beam exposure of the photoconductive region.

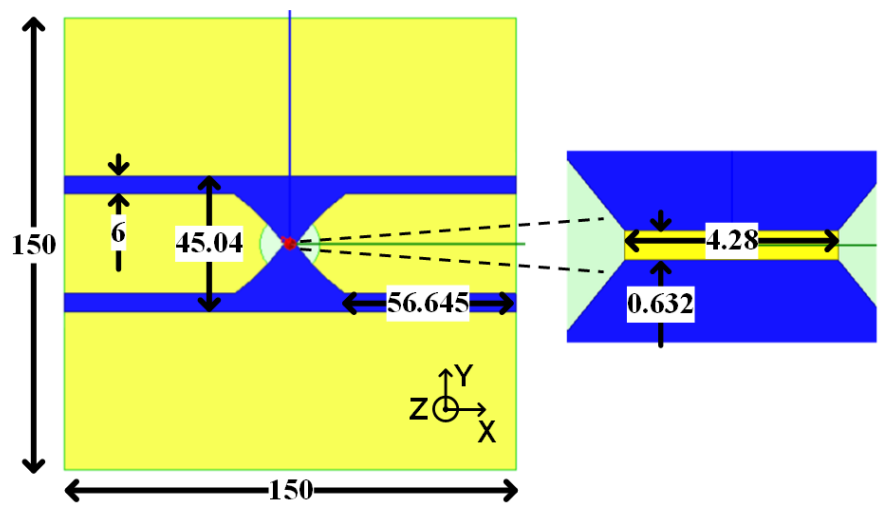

Fig. 1. Original bowtie-shaped $\mathrm{THz}$ antenna. All of the dimensions are in $\mu \mathrm{m}$.

The radiation pattern of this bowtie antenna at $1.05 \mathrm{THz}$ is shown in Fig. 2. One can see that the front-to-back ratio is $14.0 \mathrm{~dB}$. The corresponding directivity is $8.0 \mathrm{~dB}$ and the radiation efficiency is $93 \%$ at $1.05 \mathrm{THz}$. In addition, the $10 \mathrm{~dB}$ 
bandwidth of this bowtie antenna is about $0.181 \mathrm{THz}$ (from 0.996 to $1.177 \mathrm{THz}$ ). Note that since the input impedance of the photoconductive antenna is generally between 10 and 100 $\Omega$, we used $50 \Omega$ as reference impedance.

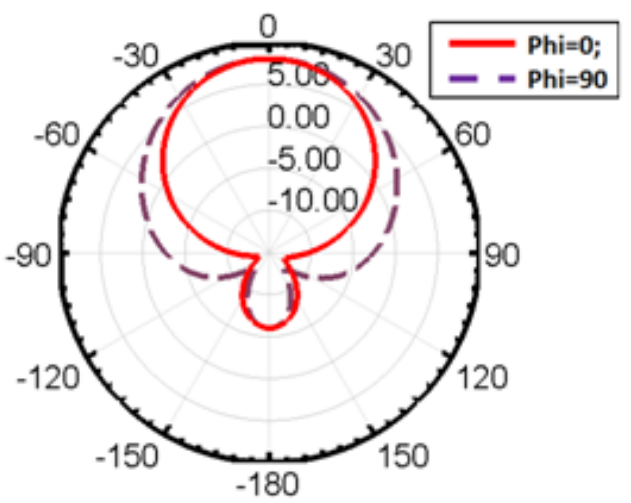

Fig. 2. Radiation pattern of the bowtie-shaped THz antenna at $1.05 \mathrm{THz}$.

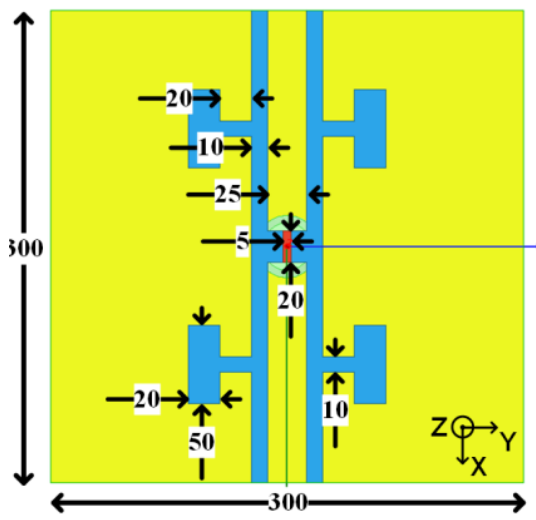

Fig. 3. Single capacitively-loaded dipole antenna design. All of the dimensions are in $\mu \mathrm{m}$.

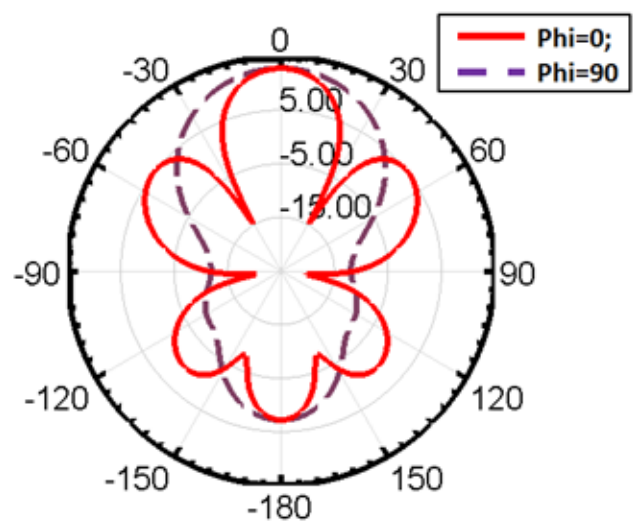

Fig. 4. Simulated radiation pattern of the single capacitively-loaded $\mathrm{THz}$ dipole antenna at $1.025 \mathrm{THz}$.

\section{Single CAPACITIVEly-LOADED DiPOLE ANTENNA DESIGN}

The single capacitively-loaded dipole antenna and its dimensions are shown in Fig. 3. The radiating element and ground plane are taken to be $0.35-\mu \mathrm{m}$-thick Ti-Au $(\sigma=1.6 \times$ $\left.10^{7} \mathrm{~S} / \mathrm{m}\right)$. Both the substrate and the superstrate were selected to be GaAs $\left(\varepsilon_{\mathrm{r}}=12.9, \mu_{\mathrm{r}}=1.0\right.$, loss tangent $\left.=0.006\right)$. The GaAs substrate and superstrate thicknesses are $59 \mu \mathrm{m}$ and 90 $\mu \mathrm{m}$, respectively, while the thickness of LTG-GaAs is $1 \mu \mathrm{m}$. The photoconductive gap region is located in the center of the figure. Because the femto-second laser beam must expose this gap region to generate the necessary electron-hole pairs, a truncated cone (frustrum) structure was cut through the ground plane and partially through the GaAs substrate to the expose the gap region. It has a $15 \mu \mathrm{m}$ upper radius, a $20 \mu \mathrm{m}$ lower radius, and $58 \mu \mathrm{m}$ height. In order to assemble several single antennas into a $\mathrm{THz}$ antenna array, the $\mathrm{DC}$ bias and ground lines are designed to connect the edges of each single antenna. The four patch-like structures on the DC bias and ground lines represent the capacitive loadings of the dipole arms; they are introduced to achieve impedance matching. It is assumed that the power level of the incident laser beam will generate an effective $50 \Omega$ source impedance. The design can be readjusted for other power levels and, hence, source impedances.

The simulation results show that the peak directivity of this single capacitively-loaded dipole antenna is $12.8 \mathrm{~dB}$ and the realized gain is $11.6 \mathrm{~dB}$ at $1.025 \mathrm{THz}$, a single-mode resonance with reasonable impedance matching. The radiation efficiency is $82 \%$ and the aperture efficiency is $144 \%$ [5]. Moreover, the $10 \mathrm{~dB}$ bandwidth of this antenna is $0.071 \mathrm{THz}$ at $1.025 \mathrm{THz}$, giving a fractional $10 \mathrm{~dB}$ bandwidth: $\mathrm{FBW}_{10 \mathrm{~dB}}=7.1 \%$. The corresponding far-field radiation pattern is shown in Fig. 4. One observes that the single $\mathrm{THz}$ antenna has a $-7.7 \mathrm{~dB}$ sidelobe level and a $10 \mathrm{~dB}$ front-to-back ratio.

\section{CAPACITIVELY-LOADED Dipole ANTENNA ARRAY DESIGN}

By connecting the DC bias and ground lines of two single capacitively-loaded $\mathrm{THz}$ dipole antennas, one can generate a two element antenna array. This antenna array was then finetuned to achieve yet better antenna performance characteristics. The structure and dimension of the fine-tuned antenna array are shown in Fig. 5. Please note that the frustum structure has been tuned to have a $25 \mu \mathrm{m}$ upper radius and a 30 $\mu \mathrm{m}$ lower radius to achieve more satisfactory radiation patterns.

The simulation results predict that the peak directivity and realized gain are both increased by approximately $2 \mathrm{~dB}$. A $77 \%$ radiation efficiency and a $120 \%$ aperture efficiency have been achieved. This aperture efficiency is slightly lower than the value for the single element system due to the increase in losses associated with the additional materials being present. Furthermore, the $10 \mathrm{~dB}$ bandwidth of the antenna array is now about $0.04 \mathrm{THz}$, which yields $\mathrm{FBW}_{10 \mathrm{~dB}}=4.0 \%$. Fig. 6 shows the simulated radiation patterns of the antenna at its resonance 
frequency, 1.0 THz. These patterns have a $-18 \mathrm{~dB}$ sidelobe level and $14 \mathrm{~dB}$ front-to-back ratio.

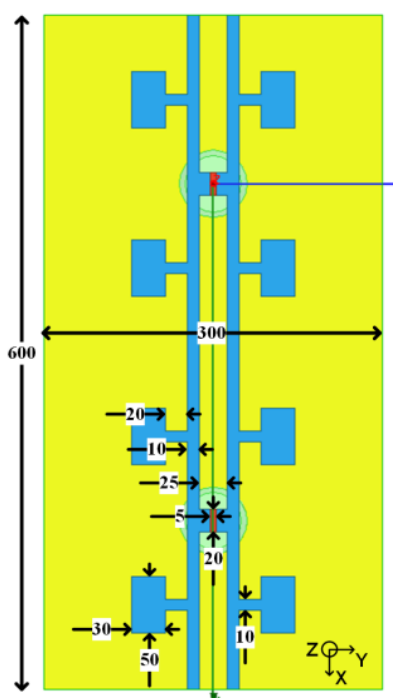

Fig. 5. The two element capacitively-loaded $\mathrm{THz}$ dipole antenna array. All of the dimensions are in $\mu \mathrm{m}$.

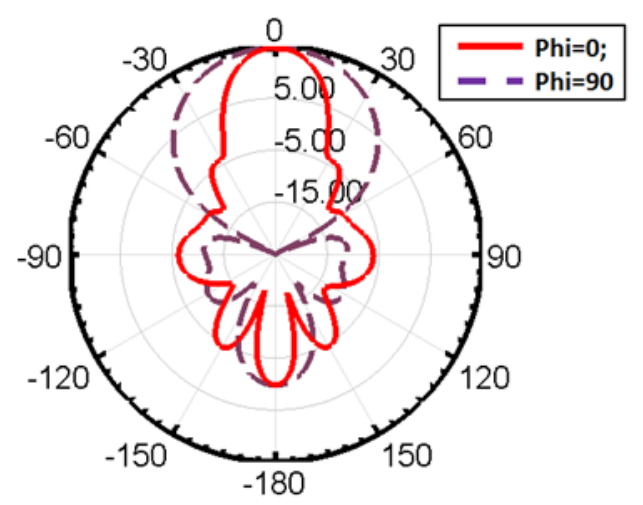

Fig. 6. Simulated radiation patterns at $1.0 \mathrm{THz}$ for the capacitively-loaded $\mathrm{THz}$ dipole antenna array.

\section{CONCLUSIONS}

In this paper, we designed several capacitively-loaded $\mathrm{THz}$ dipole-based antennas. All of these antennas were shown to achieve high directivity, resulting in over $100 \%$ aperture efficiency at resonance frequencies near $1.0 \mathrm{THz}$. It was demonstrated that a two-element array based on this single dipole element, which produced a peak directivity of $12.8 \mathrm{~dB}$ and a realized gain of $11.6 \mathrm{~dB}$ at $1.025 \mathrm{THz}$ with a radiation efficiency of $82 \%$ and an aperture efficiency is $144 \%$, achieved about another $2 \mathrm{~dB}$ increase in directivity and gain. As will be shown in the presentation, by augmenting the two-element array with a meta-film superstrate structure, the resulting antenna system was shown to have a yet further increase in its front-to-back ratio value of the array at a trade-off cost of slightly increasing the side lobe levels of its radiation patterns.

These simulation results have confirmed that the single capacitively-loaded $\mathrm{THz}$ dipole antenna will be an eligible candidate for our $\mathrm{THz}$ photoconductive antenna array. In particular, they have demonstrated that all the DC bias and ground lines can be easily connected together without impacting negatively the performance of the antenna system. A more detailed summary of these design efforts will appear in [7]. We hope to report experimental confirmation of these results in our presentation.

\section{ACKNOWLEDGMENT}

This work was supported in part by NSF contract number ECCS-1126572.

\section{REFERENCES}

[1] Z. Popovic, and E. N. Grossman, "THz metrology and instrmentation," IEEE Transaction on Terahertz Science and Technology, vol. 1, No. 1, pp. 133-144, September 2011.

[2] K. Han, T. K. Nguyen, I. Park, and H. Han, "Terahertz Yagi-Uda antenna for high input resistance," J. Infrared Milli Terahz Waves, vol. 18, pp. 441-454, 2010

[3] R. Singh, C. Rockstuhl, C. Menzel, T. P. Meyrath, M. He, H. Giessen, F. Lederer, and W. Zhang, "Spiral-type terahertz antennas and the manifestation of the Mushiake principle," Optics Express, vol. 17, pp. 9971-9980, May 2009.

[4] N. Zhu and R. W. Ziolkowski, "Progress toward THz antenna designs with high directivity and high efficiency," Proc. of the 2013 IEEE AP-S International Symposium and USNC/URSI National Radio Science Meeting, Orlando FL, paper no. 323.4, July 7-13, 2013.

[5] C. A. Balanis, Antenna Theory, 3rd Ed. New York: Wiley, 2005.

[6] R. W. Ziolkowski, "Design, fabrication, and testing of double negative metamaterials," IEEE Trans. Antennas Propag., vol. 51, no. 7, pp. 1516-1529, July 2003.

[7] N. Zhu and R. W. Ziolkowski, "Photoconductive THz antenna designs with high radiation efficiency, high directivity, and high aperture efficiency," to appear in IEEE Trans. THz Sci. Technol., 2013. 